\title{
Development of a method to maximize the transcutaneous electrical nerve stimulation intensity in women with fibromyalgia
}

This article was published in the following Dove Press journal: Journal of Pain Research

\author{
Carol GT Vance' \\ Ruth L Chimenti' \\ Dana L Dailey' \\ Katherine Hadlandsmyth ${ }^{2}$ \\ M Bridget Zimmerman ${ }^{3}$ \\ Katharine M Geasland' \\ Jonathan MWilliams ${ }^{4}$ \\ Ericka N Merriwether ${ }^{1,5}$ \\ Li Alemo Munters ${ }^{4}$ \\ Barbara A Rakel ${ }^{6}$ \\ Leslie J Crofford ${ }^{4}$ \\ Kathleen A Sluka'
}

'Department of Physical Therapy and Rehabilitation Science, The University of lowa Carver College of Medicine, lowa City, IA, USA; ${ }^{2}$ Department of Anesthesia, College of Medicine, University of lowa, lowa City, IA, USA; ${ }^{3}$ Department of Biostatistics, College of Public Health, University of lowa, lowa City, IA, USA; ${ }^{4}$ Department of Medicine/Rheumatology \& Immunology, Vanderbilt University, Nashville, TN, USA; ${ }^{5}$ Department of Physical Therapy, New York University, New York, NY, USA; ${ }^{6}$ College of Nursing, University of lowa, lowa City, IA, USA
Correspondence: Carol GT Vance Department of Physical Therapy and Rehabilitation Science, The University of lowa Carver College of Medicine, 500 Newton Road, I-252 MEB, lowa City, IA 52242, USA

Tel + I 319335979 |

Fax +l 3193359707

Email carol-vance@uiowa.edu
Introduction: Transcutaneous electrical nerve stimulation (TENS) is a non-pharmacological intervention clinically used for pain relief. The importance of utilizing the adequate stimulation intensity is well documented; however, clinical methods to achieve the highest possible intensity are not established.

Objectives: Our primary aim was to determine if exposure to the full range of clinical levels of stimulation, from sensory threshold to noxious, would result in higher final stimulation intensities. A secondary aim explored the association of pain, disease severity, and psychological variables with the ability to achieve higher final stimulation intensity.

Methods: Women with fibromyalgia $(\mathrm{N}=143)$ were recruited for a dual-site randomized controlled trial - Fibromyalgia Activity Study with TENS (FAST). TENS electrodes and stimulation were applied to the lumbar area, and intensity was increased to sensory threshold (ST), then to "strong but comfortable" ( $\mathrm{SC} 1)$, then to "noxious" $(\mathrm{N})$. This was followed by a reduction to the final stimulation intensity of "strong but comfortable" (SC2). We called this the Setting of Intensity of TENS (SIT) test.

Results: There was a significant increase from SC1 (37.5 mA IQR: 35.6-39.0) to SC2 (39.2 mA IQR: $37.1-45.3)(p<0.0001)$ with a mean increase of $1.7 \mathrm{~mA}(95 \% \mathrm{CI}: 1.5,2.2)$. Linear regression analysis showed that those with the largest increase between $\mathrm{SC} 1$ and $\mathrm{N}$ had the largest increase in SC2-SC1. Further, those with older age and higher anxiety were able to achieve greater increases in intensity (SC2-SC1) using the SIT test.

Conclusion: The SC2-SC1 increase was significantly associated with age and anxiety, with greater mean increases associated with older age and higher anxiety. Thus, although all patients may benefit from this protocol, older women and women with elevated anxiety receive the greatest benefit.

Keywords: pain, transcutaneous electrical nerve stimulation, TENS, fibromyalgia, dosage

\section{Introduction}

Pain is a universal human experience. The therapeutic reduction of pain can be addressed with pharmacological and non-pharmacological interventions. Non-pharmacological strategies are advantageous when pain is no longer protective, and the goals of patients and clinicians include avoidance of the undesirable side effects of pain medication. Transcutaneous electrical nerve stimulation (TENS) is a non-pharmacological intervention that activates the endogenous opioid system in the peripheral and central nervous systems to reduce pain. ${ }^{1,2}$ Prior randomized controlled trials (RCTs) and clinical trials have shown that TENS reduces pain, ${ }^{3-5}$ pain medication intake, and side effects, ${ }^{6-9}$ and improves function ${ }^{5,6,10-12}$ in both acute ${ }^{13}$ and chronic ${ }^{3,4}$ pain conditions. The evidence for 
the clinical use of TENS may appear conflicting. However, it is becoming increasingly evident that when TENS is dosed correctly ${ }^{8,14-16}$ and the proper outcome measures are utilized, such as pain with movement, ${ }^{5,12}$ TENS is effective.

Commercially available TENS units provide options to adjust several parameters including stimulation frequency, pulse duration, and stimulation intensity. Data show different mechanisms of action between low-frequency (LF) and highfrequency (HF) TENS, with LF TENS activating $\mu$-opioid receptors and HF TENS activating $\delta$-opioid receptors ${ }^{1,2,14,17}$ - both are effective in reducing pain when given at adequate intensities. ${ }^{3}$ There is no difference in analgesia when varying pulse duration if an adequate stimulation intensity is used. ${ }^{11}$ Conversely, the stimulation intensity is a critical variable to obtain adequate analgesia., ${ }^{3,16,18,19}$ In fact, in healthy controls, increasing intensity results in a dose-dependent hypoanalgesia ${ }^{16}$ with a strong but comfortable or the highest tolerable intensity necessary to obtain analgesia. ${ }^{16,18,19}$ Similarly in people with pain, TENS delivered at "strong but comfortable" or higher intensity is effective, whereas TENS delivered at lower intensity is ineffective..$^{5,8,11}$ Thus, the methods to achieve a greater level of stimulation intensity could improve TENS effectiveness.

Limiting factors in achieving adequate TENS intensity could be patient-related such as pain severity, disease severity, or psychological variables. In people with fibromyalgia, there is a greater sensitivity to all sensory stimuli. ${ }^{20}$ Theoretically, those with severe pain or disease could have poor tolerance to electrical stimulation. Fear of pain predicts pain threshold, but not pain tolerance in healthy controls ${ }^{21,22}$ and clinical pain intensity in patients with musculoskeletal pain. ${ }^{23}$ Increased pain catastrophizing and anxiety are associated with increased pain and reduced pain thresholds. ${ }^{24,25}$ In fact, Rakel et al previously demonstrated in an RCT for postoperative pain following total knee replacement that individuals who scored high on anxiety and pain catastrophizing had less reduction in pain with TENS than those who scored low on these psychological constructs. ${ }^{26}$ It could be that the individuals with higher anxiety and pain catastrophizing achieved a lower stimulation intensity. Thus, examining the relationships between psychological variables and the ability to achieve an adequate stimulation intensity could improve outcomes.

The primary aim of the current study was to determine whether exposure to the full range of clinical levels of stimulation (sensory threshold, strong but comfortable, and noxious) would result in higher final stimulation intensities in women with fibromyalgia. It was hypothesized that the subject's final "strong but comfortable" rating (SC2) would be greater than their initial "strong but comfortable" rating
(SC1). A secondary aim was to examine whether age, body mass index (BMI), measures of pain severity, disease severity, and psychological variables were associated with a subject's ability to achieve a higher final stimulation intensity.

\section{Materials and methods}

This is an analysis of baseline data from an ongoing clinical RCT investigating the effects of TENS in women with fibromyalgia - the Fibromyalgia Activity Study with TENS (FAST; NCT01888640). The data were collected at the first and second visits of a four-visit protocol and prior to randomization to the study intervention. This study was approved by the Human Subjects Review Board at the University of Iowa and Vanderbilt University Medical Center.

\section{Subjects}

Women diagnosed with fibromyalgia were recruited. Of the 474 women screened, 155 individuals provided written informed consent, and 143 were tested and analyzed (Figure S1). Primary reasons for exclusion were prior TENS use, pain rating $<4$, and failure to meet criteria for diagnosis of fibromyalgia. Inclusion and exclusion criteria are presented in Table S1 and were published previously. ${ }^{27}$

\section{Data collection protocol}

An initial telephonic screening was conducted in addition to a review of inclusion and exclusion criteria, prior to informed consent at the first visit. Following informed consent, subjects completed a demographic information survey. Resting pain severity was assessed with the 0-10 Numeric Rating Scale (NRS). Height and weight were recorded and then used to calculate BMI. Then, the Setting of Intensity of TENS (SIT) protocol was administered (see below). At Visit 2 (1 week following Visit 1), subjects completed the Revised Fibromyalgia Impact Questionnaire (FIQR; measure of disease severity), the Pain Catastrophizing Scale (PCS; measure of pain catastrophizing), the Patient-Reported Outcomes Measurement Information System (PROMIS) Depression module (measure of depressive symptoms), and the PROMIS Anxiety module (measure of anxious symptoms). Descriptions and psychometric properties of these measures have been presented in the previous literature from our group. ${ }^{27,28}$

\section{The SIT protocol}

Subjects were advised that a test would be conducted to check their response to TENS. This SIT test was described as being different from the TENS treatments that would follow for the remainder of the study. A standardized script 


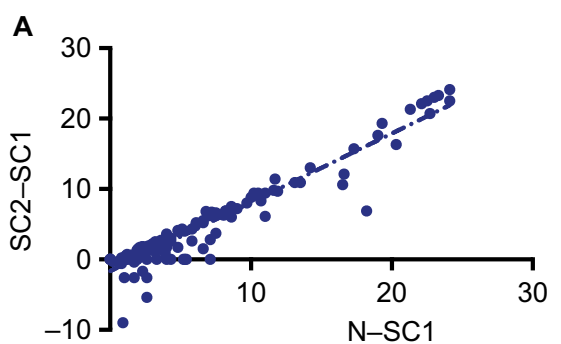

C

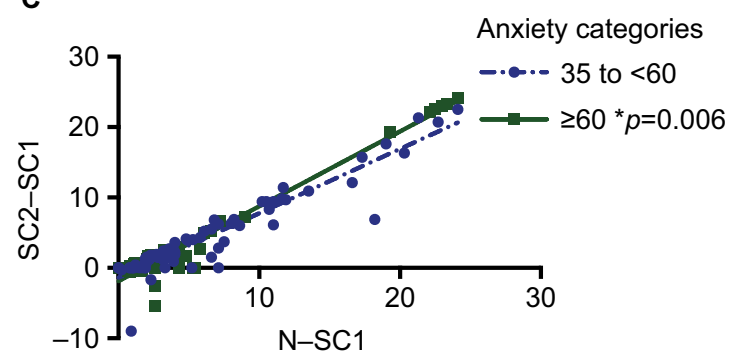

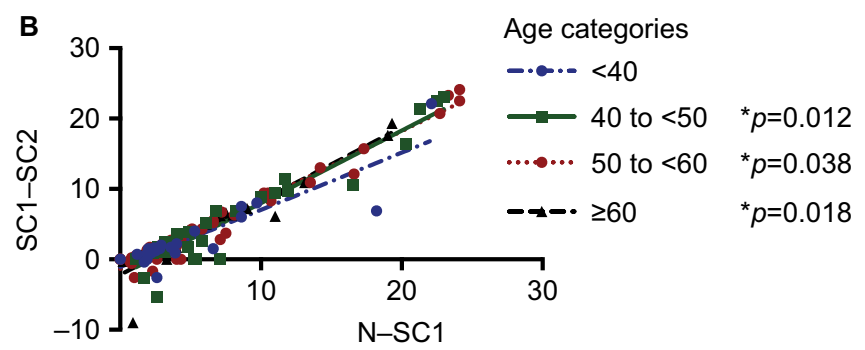

D

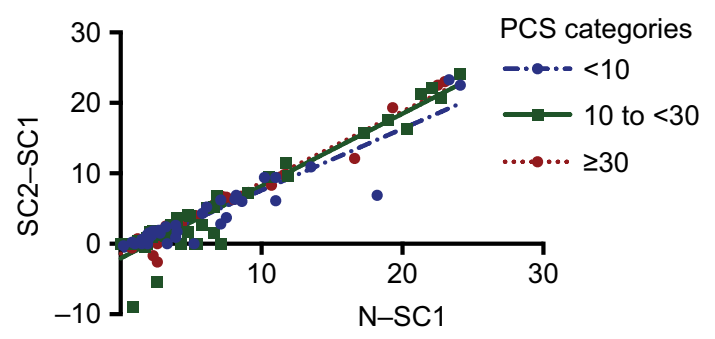

Figure I Regression of the difference in TENS intensities (mA) between "strong but comfortable I" and "strong but comfortable 2" (SC2-SCI) on the difference between "strong but comfortable I" and "noxious" for the lumbar region in (A) all subjects with fibromyalgia. Regression of the difference in TENS intensities (mA) between "strong but comfortable I" and "strong but comfortable 2" (SC2-SCI) on the difference between "strong but comfortable I" and "noxious" for the lumbar region in subjects with fibromyalgia by categories for (B) age, (C) PROMIS Anxiety with significant differences in slope, and (D) PCS with a nonsignificant difference.

Abbreviations: PCS, Pain Catastrophizing Scale; PROMIS, Patient-Reported Outcomes Measurement Information System; TENS, Transcutaneous electrical nerve stimulation.

was used to conduct the test. A large $4^{\prime \prime} \times 7^{\prime \prime}$ butterfly electrode (Empi Inc/DJO Global, Vista, CA, USA) was used to cover a greater surface area. The electrode was applied to water-cleaned skin over the lumbar paraspinal musculature between L1 and L4. The Empi Select TENS unit (Empi Inc/ DJO Global), which uses an asymmetrical biphasic alternating current, was connected to the electrode and set in the continuous mode (pulse duration $100 \mu$ s, pulse frequency $50 \mathrm{~Hz}$ ). TENS intensity was increased slowly, and subjects were instructed to indicate sensory threshold (ST); then, the intensity was increased until the sensation was perceived as "strong but comfortable" (SC1). Intensity was then increased to the point deemed "noxious" (N) by subjects, followed with a decrease in intensity until the stimulus was again characterized as "strong but comfortable" (SC2). Subjects were asked if they felt any muscle twitching at the SC2 intensity, and the examiner visually inspected or palpated the site to verify this response. Intensity (in $\mathrm{mA}$ ) was recorded at each level of stimulation. For the purpose of analyses, we defined the maximal intensity increase as $(\mathrm{N}-\mathrm{SC} 1)$ and the attained comfortable increase as (SC2-SC1).

\section{Statistical analysis}

Descriptive statistics for subject characteristics and TENS stimulation intensities ( $\mathrm{SC} 1, \mathrm{~N}$, and $\mathrm{SC} 2$ ) were calculated for the entire cohort. Validated criteria were used, when available, to define the categories of patient characteristics (Table 1). Categories for age were by decade. NRS pain scores were classified as moderate pain (4-6) and severe pain (>7). ${ }^{26}$ Categories for BMI were determined based on the accepted definition of underweight/normal $(<25)$, overweight (25-30), obese (30-35), and morbidly obese ( $>35) .{ }^{29}$ The PROMIS modules for depression and anxiety were categorized into normal and abnormally high, defined as greater than $1 \mathrm{SD}$ above the general population mean. ${ }^{30}$ The PCS was categorized based on the sample of injured workers, described in the PCS user's manual, ${ }^{31}$ population percentiles into low $(<25$ th percentile, $<10$ ), medium (26th-74th percentile, 10-30), and high $(\geq 75$ th percentile, $>30)$. Based on a pooled analysis of data from 2,000 people with fibromyalgia, the FIQR was categorized into low $(<40)$, medium $(40-60)$, and high $(>60) .{ }^{32}$ Preliminary analyses showed no relationship between opioid status and $\mathrm{SC} 2-\mathrm{SC} 1$ intensity differences, and this was not included in the analysis.

To test our first hypothesis, the Wilcoxon signed-rank test was used to compare the intensities for $\mathrm{SC} 1$ and $\mathrm{SC} 2$. To test our second aim, Pearson's correlation coefficients were calculated between TENS intensity levels and subject characteristics, using the absolute values for age, BMI, NRS pain severity, FIQR, PROMIS Depression, PROMIS Anxiety, and PCS. Linear regression - with an attained comfortable increase (SC2-SC1) as the dependent variable 
and a maximal intensity increase $(\mathrm{N}-\mathrm{SC} 1)$ as the independent variable - was conducted to estimate the mean attained comfortable increase as a function of the maximal intensity increase during the SIT test. Further linear regressions were conducted to examine the moderating effect of patient characteristics on the response to the SIT test. This was done by

Table I Descriptive statistics for demographics and subjectreported outcomes

\begin{tabular}{|c|c|}
\hline Variable & Descriptive statistics $(n=143)$ \\
\hline \multicolumn{2}{|l|}{ Age } \\
\hline Mean (SD) & $50.2(11.0)$ \\
\hline Range & $20.9-70.5$ \\
\hline \multicolumn{2}{|l|}{ BMI } \\
\hline Mean (SD) & $33.5(8.0)$ \\
\hline Range & $19.1-70.2$ \\
\hline \multicolumn{2}{|c|}{ Frequency distribution } \\
\hline$<25$ & $20(14 \%)$ \\
\hline $25 \leq 30$ & $34(24 \%)$ \\
\hline $3 \mathrm{I} \leq 40$ & $6 \mathrm{I}(43 \%)$ \\
\hline$>40$ & $28(20 \%)$ \\
\hline On opioid medication & 48 (34\%) \\
\hline \multicolumn{2}{|l|}{ NRS pain baseline } \\
\hline Median (IQR) & $6(4-7)$ \\
\hline Range & $2.5-10$ \\
\hline \multicolumn{2}{|l|}{ Frequency distribution } \\
\hline$<7$ & I 18 (83\%) \\
\hline$\geq 7$ & $25(17 \%)$ \\
\hline \multicolumn{2}{|l|}{ FIQR total } \\
\hline Mean (SD) & $56.3(17.1)$ \\
\hline Range & $21.2-93.7$ \\
\hline \multicolumn{2}{|l|}{ Frequency distribution } \\
\hline$<40$ & $29(20 \%)$ \\
\hline $40-60$ & 57 (40\%) \\
\hline$>60$ & $56(40 \%)$ \\
\hline PROMIS Depression & $(n=124)$ \\
\hline Mean (SD) & $56.6(8.5)$ \\
\hline Range & $37.1-81.1$ \\
\hline \multicolumn{2}{|l|}{ Frequency distribution } \\
\hline $35-60$ & 85 (69\%) \\
\hline$>60$ & 39 (31\%) \\
\hline PROMIS Anxiety & $(n=124)$ \\
\hline Mean (SD) & $57.6(8.2)$ \\
\hline Range & $37.1-83.1$ \\
\hline \multicolumn{2}{|l|}{ Frequency distribution } \\
\hline $35-60$ & 76 (6I\%) \\
\hline$>60$ & 48 (39\%) \\
\hline PCS & $(n=126)$ \\
\hline Mean (SD) & $20.5(13.2)$ \\
\hline Range & $0-51$ \\
\hline \multicolumn{2}{|l|}{ Frequency distribution } \\
\hline$<10$ & $36(29 \%)$ \\
\hline $10 \leq 30$ & 55 (44\%) \\
\hline$>30$ & $35(28 \%)$ \\
\hline
\end{tabular}

Abbreviations: BMI, body mass index; FIQR, the Revised Fibromyalgia Impact Questionnaire; NRS, Numeric Rating Scale; PCS, Pain Catastrophizing Scale; PROMIS, Patient-Reported Outcomes Measurement Information System. including the subject variable in the regression of attained comfortable increase with maximal intensity increase. To assess for moderating effect, an interaction effect (subject variable)*(maximum intensity increase) was included and tested in the regression model. For subject characteristics with significant moderating effects, post hoc analyses were used to compare the slopes between categories. All statistical analyses were conducted using SAS Version 9.4 statistical software (SAS Institute Inc, Cary, NC, USA).

\section{Results}

Subject demographics and clinical variables are presented in Table 1. The mean (SD) value was 50.2 (11.0) years for age and was $33.5(8.0) \mathrm{kg} / \mathrm{m}^{2}$ for BMI. Forty-eight of 143 subjects (34\%) were taking an opioid medication. For our sample, the median (IQR) for resting pain was 6 (4-7) and subjects had an average FIQR score of 56.3 (17.1), indicating that they had moderate severity of fibromyalgia symptoms. ${ }^{32}$ The average PROMIS Depression score was 56.6 (8.5) and the anxiety score was $57.6(8.2)$, with $31 \%$ and $39 \%$ presenting with depressive or anxiety symptoms, respectively. ${ }^{32}$ The mean PCS score for subjects was 20.5 (13.2), with 28\% showing moderate to severe pain catastrophizing. ${ }^{31}$ The variability in the sample size for these data is due to not all subjects completing Visit 2 because of not meeting the eligibility criteria or subject attrition.

\section{SC2 was significantly greater than SCI}

Table 2 shows the TENS intensity data for all subjects. The median TENS intensity attained was 37.5 (IQR: 35.6-39.0) $\mathrm{mA}$ for SC1, 40.4 (38.4-46.3) $\mathrm{mA}$ for $\mathrm{N}$, and 39.2 (37.1-45.3) $\mathrm{mA}$ for $\mathrm{SC} 2$, with a median maximal intensity increase of 3.5 (range 0-24.1) $\mathrm{mA}$. There was a significant increase from SC1 to SC2 $(p<0.0001)$, with a median attained comfortable increase of $1.7 \mathrm{~mA}(95 \% \mathrm{CI}: 1.5,2.2)$. There was a wide range of the individual differences in intensity between SC1 and SC2 (range -9 to $24.1 \mathrm{~mA}$ ), with $30.1 \%$ having an increase of $\geq 5 \mathrm{~mA}$ and $12.6 \%$ having an increase of $\geq 10 \mathrm{~mA}$. In addition, $24.5 \%$ of the subjects had a decrease in the TENS intensity from $\mathrm{SC} 1$ to $\mathrm{SC} 2$, ranging from 0 to $-9 \mathrm{~mA}$. The $\mathrm{SC} 1$ intensities of those who had $<5 \mathrm{~mA}$ change in SC1 to SC2 $(37.7 \pm 5.9 \mathrm{~mA})$ were similar to those that had $\mathrm{a} \geq 5 \mathrm{~mA}$ change in $\mathrm{SC} 1$ to $\mathrm{SC} 2(37.9 \pm 4.7 \mathrm{~mA})$. Linear regression to estimate the mean attained comfortable increase as a function of the maximal intensity increase (intercept: -1.65 ; slope 0.98 ) resulted in an increase between $3.2 \mathrm{~mA}(95 \% \mathrm{CI}: 2.9,3.5)$ and $21.8 \mathrm{~mA}(95 \% \mathrm{CI}: 20.8,22.7)$ for those with a maximal increase between 5 and $24 \mathrm{~mA}$, respectively (Figure 1A). 


\section{Subject variables and stimulation intensities are not correlated}

Contrary to our second aim, a direct relationship between subject variables and $\mathrm{SC} 1, \mathrm{SC} 2$, or $\mathrm{N}(p>0.05)$ was absent. There were no significant correlations between subject variables of age, BMI, pain, FIQR, PROMIS Depression, PROMIS Anxiety, or PCS and level of SC1, SC2, or N. Correlations ranged from 0.0 to 0.2 , and the upper limit of the $95 \% \mathrm{CI}$ was 0.33 (Table 3 ).

\section{Subject characteristics moderate the ability to increase the intensity of TENS after trial of noxious stimulation intensity}

Linear regression analysis to examine the moderating effect of subject characteristics on the response to the SIT test showed significant differences in slopes for the categories of age and anxious symptoms (Table 4). The older age groups (40-50, $50-60$, and $>60$ years) had a greater slope than those under

Table 2 TENS tolerance stimulation intensities (mA)

\begin{tabular}{ll}
\hline Stimulation intensity & Descriptive statistics (n= 143) \\
\hline Strong but comfortable I & $37.5(35.6-39.0)$ \\
$\quad$ Median (IQR) & $20.4-62.0$ \\
$\quad$ Range & \\
Strong but comfortable 2 & $39.2(37.1-45.3)$ \\
$\quad$ Median (IQR) & $24.8-62.0$ \\
$\quad$ Range & \\
Noxious & $40.4(38.4-46.3)$ \\
$\quad$ Median (IQR) & $24.8-62.0$ \\
Range & $1.7(0.2-6.3)$ \\
Comfortable 2-comfortable I & -9.0 to 24.I \\
$\quad$ Median (IQR) & \\
$\quad$ Range & $3.5(2.0-7.5)$ \\
Noxious-comfortable I & $0-24.1$ \\
$\quad$ Median (IQR) & Range
\end{tabular}

Abbreviation: TENS, Transcutaneous electrical nerve stimulation.
40 years (Figure 1B). Subjects in the high anxiety group had a higher slope compared to those who scored within the normal range of anxiety on the PROMIS module (Figure 1C). There was no significant moderating effect of pain catastrophizing (PCS, $p=0.057$; Figure 1D), resting pain severity $(p=0.164)$, BMI ( $p=0.616)$, disease severity (FIQR, $p=0.259)$, depressive symptoms $(p=0.222)$. (Table 4, Figure $2 \mathrm{~A}-\mathrm{D})$.

\section{Discussion}

The current study showed that exposure to the full range of TENS stimulation intensities from sensory threshold to noxious using the SIT test resulted in a greater final intensity of TENS characterized as "strong but comfortable". The study further showed that women who were older or had higher anxiety were most likely to benefit from using the SIT test, as indicated by the attained change between $\mathrm{SC} 2$ and $\mathrm{SC} 1$ being similar to the maximum possible change between $\mathrm{N}$ and $\mathrm{SC} 1$.

Whereas the current data support our hypothesis that exposing women with fibromyalgia to the full range of TENS intensities increases the final stimulation intensity, the statistically significant median change of $1.7 \mathrm{~mA}$ for the entire patient sample may not be clinically relevant. However, this difference ranged from -9 to $24 \mathrm{~mA}$, and $43 \%$ of subjects achieved an increase of at least $5 \mathrm{~mA}-$ a clinically meaningful difference in intensity. Thus, this method may be more useful for a subpopulation of individuals.

Our second aim sought to determine whether measures of pain severity, disease severity, and psychological variables were correlated with a subject's ability to achieve higher final stimulation intensity. Interestingly, the current study showed no significant correlations of the clinical level of stimulation (SC1, SC2, and $\mathrm{N}$ ) and patient characteristics. However, perceptions of stimulation are quite variable at each intensity level and are related to a number of conditions including skin characteristics such as impedance, temperature, and hydration, ${ }^{33-35}$ and neural characteristics such as sensory and

Table 3 Correlation between subject variables and TENS tolerance level

\begin{tabular}{|c|c|c|c|}
\hline \multirow[t]{2}{*}{ Variable } & \multicolumn{3}{|l|}{ Spearman's correlation $(95 \% \mathrm{Cl})$} \\
\hline & With strong but comfortable I & With strong but comfortable 2 & Noxious \\
\hline Age & $0.01(-0.16,0.18)$ & $0.04(-0.12,0.21)$ & $0.04(-0.12,0.21)$ \\
\hline BMI & $0.20(0.05,0.35)$ & $0.14(-0.03,0.30)$ & $0.15(-0.02,0.30)$ \\
\hline NRS pain baseline & $0.00(-0.17,0.17)$ & $-0.05(-0.21,0.12)$ & $-0.08(-0.20,0.08)$ \\
\hline FIQR total & $0.17(0.02,0.32)$ & $0.18(0.03,0.33)$ & $0.15(-0.00,0.30)$ \\
\hline PROMIS Depression & $0.11(-0.06,0.28)$ & $0.06(-0.11,0.23)$ & $0.06(-0.11,0.23)$ \\
\hline PROMIS Anxiety & $-0.04(-0.21,0.13)$ & $-0.07(-0.24,0.10)$ & $-0.08(-0.25,0.09)$ \\
\hline PCS & $0.00(-0.17,0.17)$ & $0.04(-0.13,0.21)$ & $-0.06(-0.23,0.11)$ \\
\hline
\end{tabular}

Abbreviations: BMI, body mass index; FIQR, the Revised Fibromyalgia Impact Questionnaire; NRS, Numeric Rating Scale; PCS, Pain Catastrophizing Scale; PROMIS, PatientReported Outcomes Measurement Information System; TENS, Transcutaneous electrical nerve stimulation. 
Table 4 Regression of attained comfortable increase (strong 2-strong I) on the expected maximum increase (noxious-strong I) Effect of age, BMI, and baseline psychometric variables

\begin{tabular}{|c|c|c|c|c|c|c|}
\hline \multirow{3}{*}{$\begin{array}{l}\text { Variable } \\
\text { Age }\end{array}$} & \multicolumn{4}{|c|}{ Linear regression parameter estimate } & \multicolumn{2}{|c|}{ Compare between groups } \\
\hline & \multicolumn{2}{|c|}{ Intercept $(95 \% \mathrm{Cl})$} & \multicolumn{2}{|c|}{ Slope $(95 \% \mathrm{Cl})$} & \multirow{2}{*}{$\begin{array}{l}\text { Intercept } \\
p=0.162\end{array}$} & \multirow{2}{*}{$\begin{array}{l}\text { Slope } \\
p=0.056\end{array}$} \\
\hline & & & & & & \\
\hline$<40$ & -1.09 & $(-2.08,-0.10)$ & 0.81 & $(0.67,0.95)$ & & \\
\hline 40 to $\leq 50$ & -2.29 & $(-3.19,-1.39)$ & 1.03 & $(0.93,1.12)$ & & 40 to $\leq 50$ vs $<40: p=0.012$ \\
\hline 50 to $\leq 60$ & -1.34 & $(-1.95,-0.73)$ & 0.98 & $(0.90,1.05)$ & & 50 to $\leq 60$ vs $<40: p=0.038$ \\
\hline$>60$ & -2.20 & $(-3.31,-1.10)$ & 1.05 & $(0.91,1.19)$ & & $>60$ vs $<40: p=0.018$ \\
\hline BMI & & & & & $p=0.603$ & $p=0.616$ \\
\hline$<25$ & -1.68 & $(-3.14,-0.23)$ & 1.01 & $(0.73,1.30)$ & & \\
\hline 25 to $\leq 30$ & -1.91 & $(-2.85,-0.96)$ & 1.02 & $(0.93,1.12)$ & & \\
\hline 30 to $\leq 40$ & -1.80 & $(-2.41,-1.19)$ & 0.94 & $(0.87,1.02)$ & & \\
\hline$>40$ & -1.10 & $(-2.07,-0.13)$ & 0.97 & $(0.86,1.08)$ & & \\
\hline NRS pain baseline & & & & & $p=0.508$ & $p=0.164$ \\
\hline$<7$ & -1.74 & $(-2.22,-1.27)$ & 0.996 & $(0.94,1.05)$ & & \\
\hline$>7$ & -1.39 & $(-2.33,-0.45)$ & 0.91 & $(0.8 \mathrm{I}, \mathrm{I} .02)$ & & \\
\hline FIQR total & & & & & $p=0.056$ & $p=0.259$ \\
\hline$<40$ & -2.61 & $(-3.67,-1.55)$ & 1.06 & $(0.91,1.21)$ & & \\
\hline $40-60$ & -1.79 & $(-2.44,-1.14)$ & 0.997 & $(0.92,1.08)$ & & \\
\hline$>60$ & -1.13 & $(-1.78,-0.48)$ & 0.937 & $(0.87,1.01)$ & & \\
\hline PROMIS Depression & & & & & $p=0.653$ & $p=0.222$ \\
\hline $35-60$ & -1.58 & $(-2.14,-1.01)$ & 0.96 & $(0.89,1.02)$ & & \\
\hline$>60$ & -1.80 & $(-2.58,-1.0 \mathrm{I})$ & 1.03 & $(0.94,1.12)$ & & \\
\hline PROMIS Anxiety & & & & & $p=0.273$ & $p=0.006$ \\
\hline $35-60$ & -1.38 & $(-1.99,-0.78)$ & 0.91 & $(0.84,0.98)$ & & \\
\hline$>60$ & -1.89 & $(-2.55,-1.22)$ & 1.06 & $(0.99,1.14)$ & & \\
\hline PCS total & & & & & $p=0.224$ & $p=0.057$ \\
\hline$<10$ & -1.16 & $(-2.04,-0.29)$ & 0.87 & $(0.77,0.98)$ & & 10 to $\leq 30$ vs. $<10: p=0.021$ \\
\hline 10 to $\leq 30$ & -2.07 & $(-2.74,-1.39)$ & 1.03 & $(0.95,1.10)$ & & $>30$ vs. $<10: p=0.063$ \\
\hline$>30$ & -1.42 & $(-2.24,-0.61)$ & 1.01 & $(0.91,1.11)$ & & $>30$ vs. 10 to $\leq 30: p=0.807$ \\
\hline
\end{tabular}

Abbreviations: BMI, body mass index; FIQR, the Revised Fibromyalgia Impact Questionnaire; NRS, Numeric Rating Scale; PCS, Pain Catastrophizing Scale; PROMIS, PatientReported Outcomes Measurement Information System.

nociceptive thresholds. ${ }^{36}$ For this reason, stimulation intensity is variable and routinely set by patient perception, making the intensity comparison between groups difficult. To achieve a common point of comparison between groups, we examined the relationship between two change scores (SC2-SC1 and $\mathrm{N}-\mathrm{SC} 1$ ). This allowed us to analyze if the SIT test would enable the subjects to approach their self-appointed maximum intensity level (noxious). We showed a strong correlation between these two measures, suggesting those with the largest difference between $\mathrm{SC} 1$ and $\mathrm{N}$ intensity were able to show the greatest increase between SC1 and SC2. Further, we showed that those with increased age and increased anxiety were able to show greater increases using the SIT test. In addition, the SIT test may be beneficial for patients who do not choose a very high initial stimulation intensity.

The current study did not show a correlation between age and sensory or noxious stimulation intensities; however, the relationship between $\mathrm{SC} 2-\mathrm{SC} 1$ and $\mathrm{N}-\mathrm{SC} 1$ did demonstrate that older subjects were better able to approach their selfappointed maximum intensity level. The association between older age and the ability to increase TENS intensity closer to their noxious level may be related to altered cutaneous sensitivity in older adults. For example, older adults show increased cutaneous pain thresholds $\mathrm{s}^{37,38}$ and higher sensory thresholds to innocuous stimulation as evidenced by the documented age-related decline in the main sensory systems of hearing, vision, taste, smell, vibration, and touch. ${ }^{39}$ When electrical stimulation was administered to healthy volunteers, the same intensities evoked lower pain ratings in the older adults (61-73 years) as compared to young adults (20-29 years). ${ }^{37}$ Similarly, the sensory perception threshold to vibration in older adults is higher than that in younger adults. ${ }^{40}$ Further, microneurography shows that the proportion of mechanosensitive $\mathrm{C}$ fibers is smaller in older (mean $=56.2$ years) adults as compared to younger adults (mean $=$ 25 years). ${ }^{41}$ These changes in sensitivity could be related to 

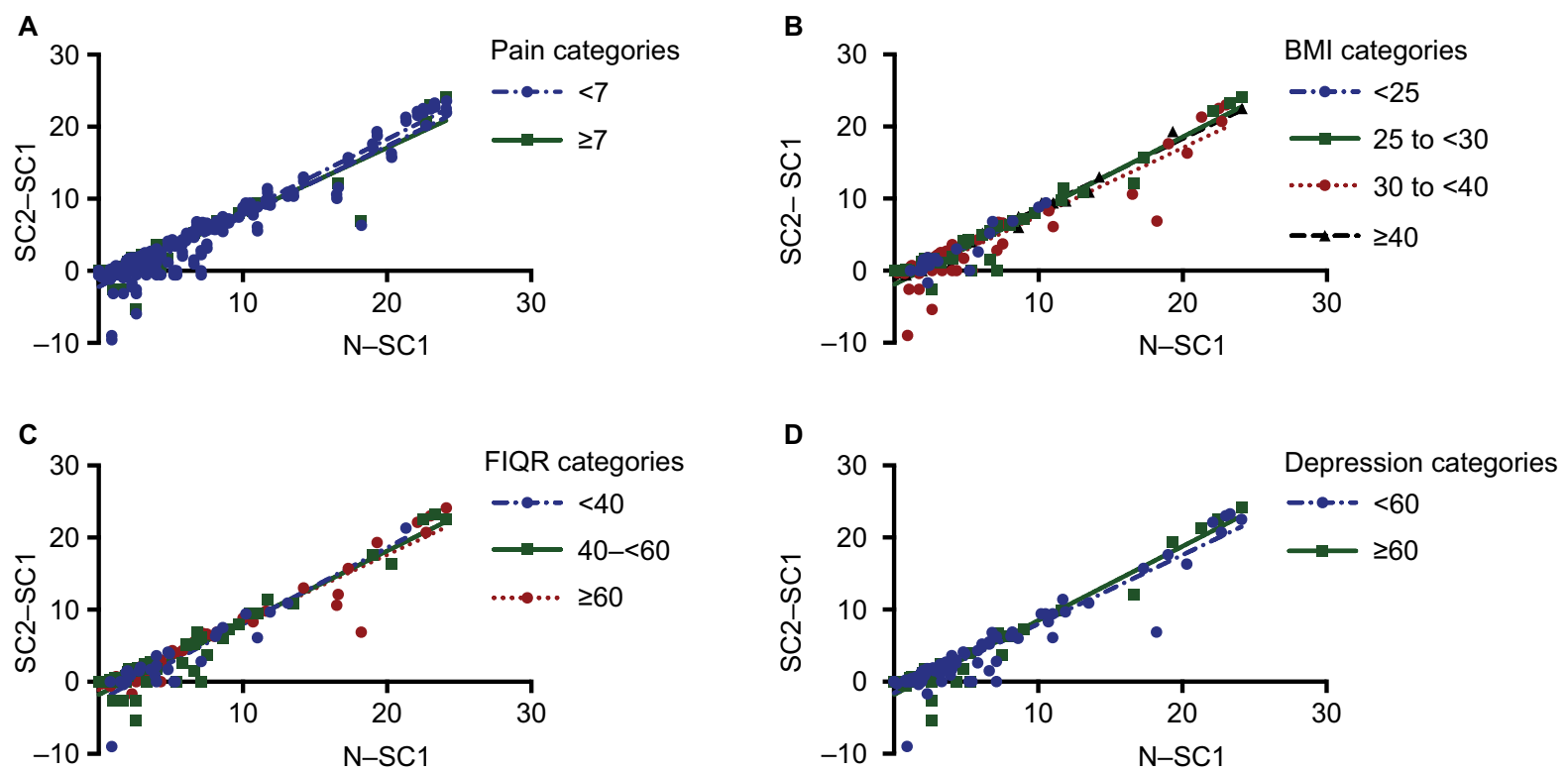

Figure 2 Regression of the difference in TENS intensities (mA) between "strong but comfortable I" and "strong but comfortable 2" (SC2-SCI) on the difference between "strong but comfortable I" and "noxious" for the lumbar region for variables with nonsignificant differences in slope between categories include (A) NRS pain at baseline, (B) BMI, (C) FIQR, and (D) PROMIS Depression

Abbreviations: BMI, body mass index; FIQR, the Revised Fibromyalgia Impact Questionnaire; NRS, Numeric Rating Scale; PROMIS, Patient-Reported Outcomes Measurement Information System; TENS, Transcutaneous electrical nerve stimulation.

alterations in cutaneous structures. Aging is associated with thinning of both the dermis and the epidermis of the skin, and thinning of the junction between these two layers, as well as alterations (up or down) in collagen production and metabolism of the skin ${ }^{42}$ - all of these factors could contribute to altered sensation. Thus, the ability of our older females to obtain a greater stimulation level of strong but comfortable (SC2) in relation to their maximal level of stimulation (N) may be related to their altered sensory processing or altered response to electrical stimulation. It should be noted, however, that stimulation intensity is difficult to interpret as it may be influenced by skin characteristics such as hydration, impedance, blood flow, and temperature. ${ }^{35,43}$

Subjects with elevated anxiety demonstrated the greatest increases in "strong but comfortable" intensity when exposed to the SIT test. Anxiety is important to assess in this population because $13 \%-64 \%$ of persons with fibromyalgia have concurrent anxiety. ${ }^{44}$ Patients with elevated anxiety may approach a new treatment, such as TENS, with caution to preemptively avoid potentially noxious stimuli due to feared harm. Rakel et al previously showed that those with increased anxiety were less responsive to TENS after total knee replacement. ${ }^{26}$ It is possible that these subjects had a lower intensity of TENS when compared to those without anxiety. As intensity is critical to effectiveness of TENS, $3,16,18,19$ these subjects could have presented with reduced effectiveness. The current study shows that those with the highest levels of anxiety displayed the greatest ability to attain SC2 intensity closest to their maximal tolerated (N) TENS intensity. The SIT test could be considered an exposure-based therapy. Exposure therapy is used to treat anxiety and aims to help people confront their fears by altering the expectancy of harm. ${ }^{45-47}$ Exposure-based techniques may be useful for those who are anxious about TENS - that is, trait anxiety - by allowing them to experience the range of non-painful stimulation. Commonly, psychological approaches are utilized in chronic pain intervention for goals of increasing activity and exercise. Non-harmful stimuli that increase the activity are used to demonstrate that the feared activity will not cause harm. ${ }^{48}$ The SIT test described in this manuscript may provide a mechanism to increase intensities of stimulation in those with high anxiety by exposing subjects to the feared stimulation and providing a non-harmful experience.

Interestingly, none of the subject characteristics assessed (age, BMI, measures of pain severity, disease severity, and psychological variables of depression, anxiety, and catastrophizing) negatively impacted the ability of subjects to achieve the greatest increase in stimulation intensity. It is possible that BMI did not impact increases in stimulation intensity because the majority of subjects were considered obese (62\%) or overweight (24\%). Overall, this suggests that clinicians can be confident in the application of the SIT test to women with 
fibromyalgia. Our overall hypothesis that exposure to the full range of electrical stimulation intensities resulted in subjects choosing a higher level is an important finding - because whereas encouraging higher stimulation intensity for pain management is desired, we find no documented methods for clinically addressing this issue. Resting pain, BMI, disease severity (FIQR), pain catastrophizing (PCS), and depression were not the predictors of the change between SC1 and SC2. This suggests that pain and its related symptoms do not directly influence the precise level of TENS stimulation intensity. It is unclear, however, what factors will be predictors of TENS effectiveness in women with fibromyalgia, or if the ability to achieve the greatest change in intensity between SC1 and SC2 is related to TENS effectiveness. Additional analysis of these subjects after completion of the clinical trial will determine the effectiveness and utility of this TENS intensity test.

\section{Acknowledgments}

This research was supported by grant nos. NIH UM1 AR063381 and NIH UM1 AR063381-S1, as well as by the CTSA award No. UL1TR000445 from the National Center for Advancing Translational Sciences to Vanderbilt University Medical Center. The sponsors had no involvement in any stage from the study design to the submission of this paper for publication.

\section{Author contributions}

CGTV, DLD, BAR, LJC, and KAS contributed to conception and design; DLD, KMG, JMW, and LAM undertook data acquisition; CGTV, RLC, KH, MBZ, ENM, LJC, and KAS conducted data analysis and interpretation; CGTV, RLC, KH, and KAS drafted the manuscript; and CGTV, RLC, DLD, KH, MBZ, KMG, JMW, ENM, LAM, BAR, LJC, and KAS critically revised the manuscript. All authors contributed toward data analysis, drafting and revising the paper and agree to be accountable for all aspects of the work.

\section{Disclosure}

The authors report no conflicts of interest in this work.

\section{References}

1. Sluka KA, Deacon M, Stibal A, Strissel S, Terpstra A. Spinal blockade of opioid receptors prevents the analgesia produced by TENS in arthritic rats. J Pharmacol Exp Ther. 1999;289(2):840-846.

2. Kalra A, Urban MO, Sluka KA. Blockade of opioid receptors in rostral ventral medulla prevents antihyperalgesia produced by transcutaneous electrical nerve stimulation (TENS). J Pharmacol Exp Ther. 2001;298(1):257-263.

3. Vance CG, Dailey DL, Rakel BA, Sluka KA. Using TENS for pain control: the state of the evidence. Pain Manag. 2014;4(3):197-209.
4. Johnson M, Martinson M. Efficacy of electrical nerve stimulation for chronic musculoskeletal pain: a meta-analysis of randomized controlled trials. Pain. 2007;130(1-2):157-165.

5. Rakel B, Frantz R. Effectiveness of transcutaneous electrical nerve stimulation on postoperative pain with movement. J Pain. 2003;4(8):455-464.

6. Freynet A, Falcoz PE. Is transcutaneous electrical nerve stimulation effective in relieving postoperative pain after thoracotomy? Interact Cardiovasc Thorac Surg. 2010;10(2):283-288.

7. Wang B, Tang J, White PF, et al. Effect of the intensity of transcutaneous acupoint electrical stimulation on the postoperative analgesic requirement. Anesth Analg. 1997;85(2):406-413.

8. Bjordal JM, Johnson MI, Ljunggreen AE. Transcutaneous electrical nerve stimulation (TENS) can reduce postoperative analgesic consumption. A meta-analysis with assessment of optimal treatment parameters for postoperative pain. Eur J Pain. 2003;7(2):181-188.

9. Lan F, Ma YH, Xue JX, Wang TL, Ma DQ. Transcutaneous electrical nerve stimulation on acupoints reduces fentanyl requirement for postoperative pain relief after total hip arthroplasty in elderly patients. Minerva Anestesiol. 2012;78(8):887-895.

10. Law PP, Cheing GL, Tsui AY. Does transcutaneous electrical nerve stimulation improve the physical performance of people with knee osteoarthritis? J Clin Rheumatol. 2004;10(6):295-299.

11. Sluka KA, Bjordal JM, Marchand S, Rakel BA. What makes transcutaneous electrical nerve stimulation work? Making sense of the mixed results in the clinical literature. Phys Ther. 2013;93(10):1397-1402.

12. Dailey DL, Rakel BA, Vance CG, et al. Transcutaneous electrical nerve stimulation reduces pain, fatigue and hyperalgesia while restoring central inhibition in primary fibromyalgia. Pain. 2013;154(11):2554-2562.

13. Sbruzzi G, Silveira SA, Silva DV, Coronel CC, Plentz RD. Transcutaneous electrical nerve stimulation after thoracic surgery: systematic review and meta-analysis of 11 randomized trials. Rev Bras Cir Cardiovasc. 2012;27(1):75-87.

14. Léonard G, Cloutier C, Marchand S. Reduced analgesic effect of acupuncture-like TENS but not conventional TENS in opioid-treated patients. J Pain. 2011;12(2):213-221.

15. Liebano RE, Rakel B, Vance CG, Walsh DM, Sluka KA. An investigation of the development of analgesic tolerance to TENS in humans. Pain. 2011;152(2):335-342.

16. Moran F, Leonard T, Hawthorne S, et al. Hypoalgesia in response to transcutaneous electrical nerve stimulation (TENS) depends on stimulation intensity. J Pain. 2011;12(8):929-935.

17. Leonard G, Goffaux P, Marchand S. Deciphering the role of endogenous opioids in high-frequency TENS using low and high doses of naloxone. Pain. 2010;151(1):215-219.

18. Pantaleão MA, Laurino MF, Gallego NL, et al. Adjusting pulse amplitude during transcutaneous electrical nerve stimulation (TENS) application produces greater hypoalgesia. J Pain. 2011;12(5):581-590.

19. Rakel B, Cooper N, Adams HJ, et al. A new transient sham TENS device allows for investigator blinding while delivering a true placebo treatment. J Pain. 2010;11(3):230-238.

20. Geisser ME, Glass JM, Rajcevska LD, et al. A psychophysical study of auditory and pressure sensitivity in patients with fibromyalgia and healthy controls. J Pain. 2008;9(5):417-422.

21. Lee JE, Watson D, Frey-Law LA. Psychological factors predict local and referred experimental muscle pain: a cluster analysis in healthy adults. Eur J Pain. 2013;17(6):903-915.

22. George SZ, Dannecker EA, Robinson ME. Fear of pain, not pain catastrophizing, predicts acute pain intensity, but neither factor predicts tolerance or blood pressure reactivity: an experimental investigation in pain-free individuals. Eur J Pain. 2006;10(5):457-465.

23. George SZ, Hirsh AT. Psychologic influence on experimental pain sensitivity and clinical pain intensity for patients with shoulder pain. $J$ Pain. 2009;10(3):293-299.

24. Block PR, Thorn BE, Kapoor S, White J. Pain catastrophizing, rather than vital signs, associated with pain intensity in patients presenting to the Emergency Department for Pain. Pain Manag Nurs. 2017;18(2):102-109. 
25. Kjøgx H, Kasch H, Zachariae R, Svensson P, Jensen TS, Vase L. Experimental manipulations of pain catastrophizing influence pain levels in patients with chronic pain and healthy volunteers. Pain. 2016;157(6):1287-1296.

26. Rakel BA, Zimmerman MB, Geasland K, et al. Transcutaneous electrical nerve stimulation for the control of pain during rehabilitation after total knee arthroplasty: a randomized, blinded, placebo-controlled trial. Pain. 2014;155(12):2599-2611.

27. Noehren B, Dailey DL, Rakel BA, et al. Effect of transcutaneous electrical nerve stimulation on pain, function, and quality of life in fibromyalgia: a double-blind randomized clinical trial. Phys Ther. 2015;95(1):129-140.

28. Merriwether EN, Rakel BA, Zimmerman MB, et al. Reliability and construct validity of the Patient-Reported Outcomes Measurement Information System (PROMIS) instruments in women with fibromyalgia. Pain Med. 2017;18(8):1485-1495.

29. Jensen MD, Ryan DH, Apovian CM, et al; American College of Cardiology/American Heart Association Task Force on Practice Guidelines; Obesity Society. 2013 AHA/ACC/TOS guideline for the management of overweight and obesity in adults: a report of the American College of Cardiology/American Heart Association Task Force on Practice Guidelines and The Obesity Society. Circulation. 2014;129(25 Suppl 2):S102-S138

30. Patient-Reported Outcome Measurement Information System. Available from: https://www.assessmentcenter.net/documents/PROMIS\%20 [Anxiety]\%20Scoring\%20Manual.pdf. Accessed August 30, 2018.

31. Sullivan MJL. The Pain Catastrophizing Scale. User Manual. Montreal, QC: McGill University; 1995:22.

32. Bennett RM, Bushmakin AG, Cappelleri JC, Zlateva G, Sadosky AB Minimal clinically important difference in the fibromyalgia impact questionnaire. J Rheumatol. 2009;36(6):1304-1311.

33. Maetzler W, Klenk J, Becker C, Zscheile J, Gabor KS, Lindemann U. Longitudinal changes of nerve conduction velocity, distal motor latency, compound motor action potential duration, and skin temperature during prolonged exposure to cold in a climate chamber. Int $J$ Neurosci. 2012;122(9):528-531.

34. Lévêque JL, Dresler J, Ribot-Ciscar E, Roll JP, Poelman C. Changes in tactile spatial discrimination and cutaneous coding properties by skin hydration in the elderly. J Invest Dermatol. 2000;115(3):454-458.
35. Vance CG, Rakel BA, Dailey DL, Sluka KA. Skin impedance is not a factor in transcutaneous electrical nerve stimulation effectiveness. $J$ Pain Res. 2015;8:571-580.

36. Boada MD, Eisenach JC, Ririe DG. Mechanical sensibility of nociceptive and non-nociceptive fast-conducting afferents is modulated by skin temperature. J Neurophysiol. 2016;115(1):546-553.

37. Kemp J, Després O, Pebayle T, Dufour A. Age-related decrease in sensitivity to electrical stimulation is unrelated to skin conductance: an evoked potentials study. Clin Neurophysiol. 2014;125(3):602-607.

38. Chakour MC, Gibson SJ, Bradbeer M, Helme RD. The effect of age on A delta- and C-fibre thermal pain perception. Pain. 1996;64(1):143-152.

39. Humes LE, Busey TA, Craig JC, Kewley-Port D. The effects of age on sensory thresholds and temporal gap detection in hearing, vision, and touch. Atten Percept Psychophys. 2009;71(4):860-871.

40. Stuart M, Turman AB, Shaw J, Walsh N, Nguyen V. Effects of aging on vibration detection thresholds at various body regions. BMC Geriatr. 2003;3:1.

41. Namer B, Barta B, Ørstavik K, et al. Microneurographic assessment of C-fibre function in aged healthy subjects. $J$ Physiol. 2009;587(2):419-428.

42. Calleja-Agius J, Brincat M, Borg M. Skin connective tissue and ageing. Best Pract Res Clin Obstet Gynaecol. 2013;27(5):727-740.

43. Cramp FL, McCullough GR, Lowe AS, Walsh DM. Transcutaneous electric nerve stimulation: the effect of intensity on local and distal cutaneous blood flow and skin temperature in healthy subjects. Arch Phys Med Rehabil. 2002;83(1):5-9.

44. Fietta P, Fietta P, Manganelli P. Fibromyalgia and psychiatric disorders. Acta Biomed. 2007;78(2):88-95.

45. Hofmann SG. Cognitive processes during fear acquisition and extinction in animals and humans: implications for exposure therapy of anxiety disorders. Clin Psychol Rev. 2008;28(2):199-210.

46. Vlaeyen JW, Linton SJ. Fear-avoidance and its consequences in chronic musculoskeletal pain: a state of the art. Pain. 2000;85(3):317-332.

47. Vlaeyen JW, Linton SJ. Fear-avoidance model of chronic musculoskeletal pain: 12 years on. Pain. 2012;153(6):1144-1147.

48. Ehde DM, Dillworth TM, Turner JA. Cognitive-behavioral therapy for individuals with chronic pain: efficacy, innovations, and directions for research. Am Psychol. 2014;69(2):153-166. 


\section{Supplementary materials}

Screened $\mathrm{N}=474$

Excluded $\mathrm{N}=203$

Failed inclusion criteria $\mathrm{N}=41$

Declined $\mathrm{N}=40$

Lost $\mathrm{N}=35$

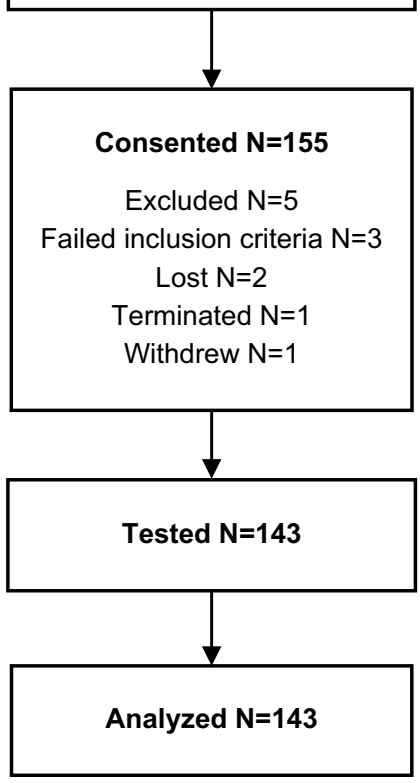

Figure SI Subject recruitment.

Journal of Pain Research

\section{Publish your work in this journal}

The Journal of Pain Research is an international, peer reviewed, open access, online journal that welcomes laboratory and clinical findings in the fields of pain research and the prevention and management of pain. Original research, reviews, symposium reports, hypothesis formation and commentaries are all considered for publication.
Table SI Inclusion and exclusion criteria

Inclusion criteria:

- Women between 18 and 70 years of age

- Diagnosis of fibromyalgia by the 1990 ACR criteria (1 I//8 tender points)

- History of cervical or lumbar pain with fibromyalgia (this is expected in all patients because axial pain is required for diagnosis)

- Current stable treatment regimen for the last 4 weeks and projected stable treatment regimen for the next 2 months.

- English speaking

Exclusion criteria:

- Current disease or a history of cardiovascular, pulmonary, neurological, endocrine, or renal disease that would preclude the involvement in the study.

- TENS use in the last 5 years

- Pacemaker

- Uncontrolled blood pressure or diabetes

- Neuropathic pain condition

- Systemic autoimmune disorder (lupus, PMR, RA, psoriasis, psoriatic arthritis)

- Spinal fusion - cervical or lumbar

- Metal implants in the spine

- Severe skin allergy to adhesives

- Allergy to nickel or adhesives

- Pain level $<4$

- Pregnancy

- Epilepsy

- Change in or new drug or treatment program within the last month or in the next 2 months - that is, must have a stable treatment plan

Abbreviations: ACT, American College of Rheumatology; PMR, polymyalgia rheumatica; RA, rheumatoid arthritis; TENS, Transcutaneous electrical nerve stimulation.
The manuscript management system is completely online and includes a very quick and fair peer-review system, which is all easy to use. Visit http://www.dovepress.com/testimonials.php to read real quotes from published authors. 